\title{
Maternal dietary patterns and gestational diabetes mellitus: a large prospective cohort study in China
}

\author{
Jian-Rong $\mathrm{He}^{1} \dagger$, Ming-Yang Yuan ${ }^{1} \dagger$, Nian-Nian $\mathrm{Chen}^{1}$, Jin-Hua $\mathrm{Lu}^{1}$, Cui-Yue $\mathrm{Hu}^{1}$, Wei-Bi Mai ${ }^{2}$, \\ Rui-Fang Zhang ${ }^{2}$, Yong-Hong Pan $^{2}$, Lan Qiu ${ }^{1}$, Ying-Fang Wu ${ }^{1}$, Wan-Qing Xiao ${ }^{1}$, Yu Liu ${ }^{1}$, \\ Hui-Min $\mathrm{Xia}^{1 *}$ and Xiu Qiu ${ }^{1 *}$ \\ ${ }^{1}$ Division of Birth Cohort Study, Guangzhou Women and Children's Medical Center, Guangzhou Medical University, \\ 9 Junsui Road, Tianhe District, Guangzhou 510623, People's Republic of China \\ ${ }^{2}$ Department of Obstetrics and Gynecology, Guangzhou Women and Children's Medical Center, \\ Guangzhou Medical University, Guangzhou, People's Republic of China
}

(Submitted 1 November 2014 - Final revision received 29 January 2015 - Accepted 11 February 2015 - First published online 30 March 2015)

\begin{abstract}
Few studies have explored the relationship between dietary patterns and the risk of gestational diabetes mellitus (GDM). Evidence from non-Western areas is particularly lacking. In the present study, we aimed to examine the associations between dietary patterns and the risk of GDM in a Chinese population. A total of 3063 pregnant Chinese women from an ongoing prospective cohort study were included. Data on dietary intake were collected using a FFQ at 24-27 weeks of gestation. GDM was diagnosed using a $75 \mathrm{~g}, 2 \mathrm{~h}$ oral glucose tolerance test. Dietary patterns were determined by principal components factor analysis. A log-binomial regression model was used to examine the associations between dietary pattern and the risk of GDM. The analysis identified four dietary patterns: vegetable pattern; protein-rich pattern; prudent pattern; sweets and seafood pattern. Multivariate analysis showed that the highest tertile of the vegetable pattern was associated with a decreased risk of GDM (relative risk (RR) 0.79, $95 \%$ CI 0.64, 0.97), compared with the lowest tertile, whereas the highest tertile of the sweets and seafood pattern was associated with an increased risk of GDM (RR 1.23, 95\% CI 1.02, 1.49). No significant association was found for either the protein-rich or the prudent pattern. The protective effect of a high vegetable pattern score was more evident among women who had a family history of diabetes $(P$ for interaction $=0.022)$. These findings suggest that the vegetable pattern was associated with a decreased risk of GDM, while the sweets and seafood pattern was associated with an increased risk of GDM. These findings may be useful in dietary counselling during pregnancy.
\end{abstract}

Key words: Dietary patterns: Gestational diabetes mellitus: Prospective studies: China

Gestational diabetes mellitus (GDM), defined as glucose intolerance with onset or first recognition during pregnancy, is one of the most common pregnancy complications, and has adverse health effects on both mothers and offspring ${ }^{(1)}$. Women with GDM have an elevated risk of hypertensive disorders and type 2 diabetes after delivery ${ }^{(1,2)}$, while offspring of women with GDM have an increased risk of obesity, the metabolic syndrome, and diabetes later in life ${ }^{(1,2)}$. In addition, epidemiological studies have provided evidence that the incidence of GDM is rising over time ${ }^{(3,4)}$, although consensus on a universal standard for diagnosis has not been reached ${ }^{(5)}$. Therefore, there is an urgent need to identify risk factors for the prevention of GDM.
As a modifiable factor, dietary habits have been shown to contribute to the development of type 2 diabetes, while research into the relationship between dietary factors and GDM is just emerging ${ }^{(6)}$. For instance, several cross-sectional or retrospective studies have shown that high intakes of saturated fat, cholesterol or eggs are associated with an increased risk of $\mathrm{GDM}^{(7-9)}$, and polyunsaturated fat intake appears to have a protective effect ${ }^{(7,10)}$. In contrast, another study has found no association between the risk of GDM and intakes of total dietary, saturated and polyunsaturated fat ${ }^{(11)}$. In addition, high glycaemic load has been reported to increase the risk of $\mathrm{GDM}^{(12)}$. In addition, a recent prospective study has found that high intakes of animal protein increased the

Abbreviations: BIGCS, Born in Guangzhou Cohort Study; GDM, gestational diabetes mellitus; GWCMC, Guangzhou Women and Children's Medical Center; OGTT, oral glucose tolerance test; RR, relative risk.

*Corresponding authors: H.-M. Xia, fax +8620 38076001, email huimin.xia876001@gmail.com; X. Qiu, fax +86 20 38076639, email qxiu0161@163.com † Both authors contributed equally to this work. 
risk of GDM, while high intakes of vegetable protein decreased the risk ${ }^{(13)}$.

Most previous studies exploring the effects of dietary factors on the risk of GDM have focused on individual nutrients or food items ${ }^{(7-12)}$. However, individual foods contain numerous nutrients, and foods are not consumed in isolation; thus, studying a single nutrient or food item cannot account for the complex interactions of multiple nutrients. In contrast, dietary pattern analysis could present a broader view of food and nutrient consumption, and examine the effects of overall diet on health ${ }^{(14)}$. Nevertheless, only a few studies in Western countries have examined the effects of dietary patterns on the risk of $\mathrm{GDM}^{(11,15-17)}$. Furthermore, to our knowledge, there is a lack of studies on Asian populations, who have distinct dietary habits. In the present study, we used data from a large prospective cohort study to examine the associations between major dietary patterns and the risk of GDM in a Chinese population.

\section{Materials and methods}

\section{Design}

The present study was embedded in the Born in Guangzhou Cohort Study (BIGCS), an ongoing collaboration between the Guangzhou Women and Children's Medical Center (GWCMC), China and the University of Birmingham, UK. This prospective study commenced in February 2012 and aims to investigate short- and long-term health consequences of environmental risk factors in the next generation in China, whose parents have experienced rapid societal transitions in their lifetime.

The sampling frame only included pregnant women attending their first routine antenatal examinations (usually about week 16) at two campuses of the GWCMC, which is the largest of its kind in southern China with patronage throughout the municipality of Guangzhou and its vicinity, handling $12000-$ 15000 deliveries annually (out of a total of 220000 births in the entire municipality). However, only those who resided within Guangzhou and intended to remain in Guangzhou with their child for $\geq 3$ years were considered eligible and were invited to participate in the BIGCS.

Maternal exposure was assessed using self-completed questionnaires. The first (Q1), second (Q2) and third (Q3) questionnaires were administered at recruitment and at gestational ages of 24-27 and 35-38 weeks, respectively; the questionnaires acquired demographic and socio-economic information, as well as data on exposures in the workplace and at home, personal lifestyle, medical history, and health status before and during pregnancy. The protocols of the BIGCS were approved by the Institutional Ethics Committee of the GWCMC. Written informed consent was obtained from all participants.

For the present study, we used data collected from pregnant women recruited between February 2012 and February 2014. During this period, we approached 9494 pregnant women attending their first routine antenatal examinations, and $94.9 \%$ were eligible; among those who were eligible, a total of $6252(69 \cdot 4 \%)$ agreed to participate in the BIGCS, while $2758(30.6 \%)$ refused. Of the women recruited, we excluded those who had a history of previous diabetes (type 1 or type 2 ) or hypertension ( $n$ 15), had pregnancy terminations or stillbirths ( $n$ 49), had multiple gestation ( $n$ 114), had missing ( $n$ 351) or incomplete ( $n$ 107) records on oral glucose tolerance test (OGTT), had an incomplete dietary questionnaire ( $n$ 1803), completed the dietary questionnaire after the OGTT ( $n$ 563), had missing ( $n$ 11) or implausible ( $n$ 17) data on food items, or dropped out before the OGTT ( $n$ 159), resulting in a final total of 3063 women in the present analysis. The comparisons of the characteristics between women who took part in the BIGCS and those who were eligible but did not participate are presented in online Supplementary Table S1. The age distributions in the two groups were similar, while educational level was higher among women who participated. Women who were included in the present study tended to be slightly older and nulliparous and had a higher education and income level than those who were not included.

\section{Assessment of diet and covariates}

Dietary intake was assessed using a self-administered FFQ at the Q2 interview. Participants were asked about the frequency of consumption of sixty-four specified food items as well as additional questions about soup, beverages and cooking oil. For each food item, participants reported how many times they had consumed it 'in the past week'. Data on the amount consumed were not collected. Individual food items were combined into twenty-four groups according to a similar nutrient profile or culinary uses. The frequencies of intake of food groups were calculated by summing the weekly consumption frequencies of grouped foods, assuming that different food items contributed equally in the same group. Some food items were classified individually if the nutrient content was distinctly different (e.g. eggs or nuts) or if they were suspected to represent distinct dietary patterns (e.g. mushrooms or seaweed) ${ }^{(16)}$. The validity and reproducibility of this FFQ was evaluated based on a subsample of cohort participants. In the validation study, a total of 210 women completed (1) the first FFQ (FFQ1) at 24-27 weeks of gestation, (2) three $24 \mathrm{~h}$ dietary recalls, including two weekdays and one weekend, once per week during weeks 29-31 of gestation, and (3) the second FFQ (FFQ2) at 33-35 weeks of gestation. The crude Spearman correlation coefficients for consumption frequencies of food groups between the FFQ1 and the FFQ2 ranged from 0.33 (sea vegetable) to 0.71 (soup). For the FFQ2 and the $24 \mathrm{~h}$ dietary recalls, the crude Spearman correlation coefficients for consumption frequencies of food groups ranged from 0.23 (preserved vegetable) to 0.62 (dairy products).

Participants who completed the FFQ after the OGTT were excluded because their diet may have changed after the OGTT. Additional information collected from the FFQ1 included maternal age at recruitment, education level (middle school or below, college, undergraduate, or postgraduate), monthly income $(<1500,1500-4500,4501-9000$ 
or $\geq 9001$ yuan, where 1 yuan $=0 \cdot 16$ US cents), parity, prepregnancy maternal height and weight, and history of diabetes (first-degree relatives). Pre-pregnancy BMI $\left(\mathrm{kg} / \mathrm{m}^{2}\right)$ was calculated as the ratio of weight $(\mathrm{kg})$ to squared height $\left(\mathrm{m}^{2}\right)$, and was categorised into three groups $(<18.5,18.5-23.9$ and $\left.\geq 24 \mathrm{~kg} / \mathrm{m}^{2}\right)^{(18)}$

\section{Glucose tolerance outcomes}

Participants were routinely given a $75 \mathrm{~g}, 2 \mathrm{~h}$ OGTT for GDM screening at a prenatal care visit. The diagnosis of GDM was made when any of the following blood glucose values was met or exceeded based on the criteria developed by the International Association of Diabetes and Pregnancy Study Groups ${ }^{(19)}$ : fasting, $5 \cdot 1 \mathrm{mmol} / 1 ; 1 \mathrm{~h}, 10 \cdot 0 \mathrm{mmol} / 1 ; 2 \mathrm{~h}$, $8.5 \mathrm{mmol} / \mathrm{l}$.

\section{Statistical analysis}

The principal components factor analysis with orthogonal (varimax) rotation was used to derive the dietary patterns. To reduce the influence of food groups with a higher frequency and greater variance, the consumption frequencies of each food group were standardised according to the mean and standard deviation before extraction of dietary patterns ${ }^{(20)}$. We retained four factors (dietary patterns) based on an inspection of scree plots and the interpretability of the results. Each dietary pattern was formed using linear combinations of food groups. The factor loadings, namely coefficients defining these linear combinations, represent the correlations of each food group with the corresponding dietary pattern. Food groups with loadings $> \pm 0.25$ were selected to describe the dietary patterns. We calculated factor scores for each dietary pattern by summing the consumption frequencies of each food group, weighted by their factor loadings for each participant. These scores were used as exposure variables for further analysis. Differences in maternal characteristics among groups were tested by using $t$ tests or ANOVA for continuous variables or $\chi^{2}$ tests for categorical variables. The analyses were performed using SPSS software version 17.0 (SPSS, Inc.).

A log-binomial regression model was used to examine the associations between dietary pattern scores and the risk of GDM, adjusting for maternal age, education level, monthly income, parity, pre-pregnancy BMI and family history of diabetes. Relative risks (RR) with their corresponding 95\% CI were calculated using PROC GENMOD in SAS statistical software version 9.2 (SAS Institute, Inc.) ${ }^{(21,22)}$.

The dietary pattern scores were included in the model as tertiles (tertile 1-tertile 3). All four dietary patterns were simultaneously entered into the models. $P$ for trend was evaluated using the median score for each category of dietary patterns and analysed as a continuous variable in multivariate models. We also performed stratified analysis to examine whether the effect of dietary patterns differed across maternal characteristics, including maternal age $(<35 v$. $\geq 35$ years $)$, education level (middle school or below $v$. college, undergraduate and postgraduate), monthly income $(<1500 v$.
$1500-4500,4501-9000$ and $\geq 9001$ yuan), pre-pregnancy BMI $\left(<18.5 v \cdot 18.5-23.9\right.$ and $\geq 24 \mathrm{~kg} / \mathrm{m}^{2}$ ), family history of diabetes (no $v$. yes) and parity $(0 v, \geq 1)$. The interaction between the dietary patterns and maternal characteristics was evaluated by including multiplicative interaction terms in multivariate models.

A two-tailed $P$ value $<0.05$ was considered statistically significant.

\section{Results}

\section{Dietary patterns}

In the present study, four main dietary patterns were extracted from the factor analysis. The factor loadings for each dietary pattern are listed in Table 1 . The first pattern indicated the vegetable pattern and explained $12 \cdot 2 \%$ of the total variance of food intake. It was characterised by frequent intake of root vegetables, beans, mushrooms, melon vegetables, seaweed, other legumes, fruits, leafy and cruciferous vegetables, processed vegetables, nuts, and cooking oil. The second pattern indicated the protein-rich pattern and explained $8.2 \%$ of the total variance. It was characterised by frequent intake of poultry, red meat, animal organ meat, grains (mainly refined), processed meat, fish, soups, leafy and cruciferous vegetables, and eggs. The third pattern indicated the prudent pattern and explained $6.7 \%$ of the total variance. It was characterised by frequent intake of dairy products, nuts, eggs, fish, soups, fruits, and infrequent intake of processed meat, sugarsweetened beverages, and processed vegetables. The fourth pattern indicated the sweets and seafood pattern and explained $5 \cdot 8 \%$ of the total variance. It was characterised by frequent intake of Cantonese desserts, molluscs and shellfish, and sugar-sweetened beverages and low intakes of grains (mainly refined) and leafy and cruciferous vegetables. All the four patterns together accounted for $32.9 \%$ of the total variation (Table 1). There were $1259(41 \cdot 1 \%)$ and 1224 (40.0\%) women who were categorised into the highest and lowest tertiles for more than one pattern, respectively.

\section{Participant characteristics}

The characteristics of the study participants are presented in Table 2. The mean age was 28.9 (SD 3.2) years. In addition, $53.4 \%$ of the participants had an education level of undergraduate, $40 \cdot 4 \%$ reported a monthly income of 4501-9000 yuan, $8.8 \%$ were overweight or obese (pre-pregnancy BMI $\left.\geq 24 \mathrm{~kg} / \mathrm{m}^{2}\right), 19 \cdot 4 \%$ had a family history of diabetes, and most (93.3\%) were nulliparous (Table 2 ).

\section{Participant characteristics based on dietary pattern scores}

The distributions of the baseline characteristics according to tertiles of dietary pattern scores are presented in Table 2. Women with a high vegetable pattern score tended to have a higher education and income level, while those with a high protein-rich pattern score were more likely to be younger and parous and have less education level (Table 2). 
Table 1. Factor loadings for the four dietary patterns identified by the principal components factor analysis in 3063 pregnant women from the Born in Guangzhou Cohort Study*

\begin{tabular}{|c|c|c|c|}
\hline Dietary patterns & Food & $\begin{array}{l}\text { Factor loading } \\
\text { coefficient }\end{array}$ & $\begin{array}{l}\text { Cumulative variance } \\
\text { explained }(\%)\end{array}$ \\
\hline \multirow[t]{11}{*}{ Vegetable } & Root vegetables & 0.63 & $12 \cdot 2$ \\
\hline & Beans & 0.63 & \\
\hline & Mushrooms & 0.61 & \\
\hline & Melon vegetables & 0.59 & \\
\hline & Seaweed & 0.56 & \\
\hline & Other legumes & 0.49 & \\
\hline & Fruits & 0.43 & \\
\hline & Leafy and cruciferous vegetables & 0.34 & \\
\hline & Processed vegetables & 0.34 & \\
\hline & Nuts & 0.32 & \\
\hline & Cooking oil & 0.25 & \\
\hline \multirow[t]{9}{*}{ Protein-rich } & Poultry & 0.62 & $20 \cdot 3$ \\
\hline & Red meat & 0.49 & \\
\hline & Animal organ meat & 0.48 & \\
\hline & Grains & 0.48 & \\
\hline & Processed meat & 0.41 & \\
\hline & Fish & 0.36 & \\
\hline & Soups & 0.34 & \\
\hline & Leafy and cruciferous vegetables & 0.33 & \\
\hline & Eggs & 0.30 & \\
\hline \multirow[t]{9}{*}{ Prudent } & Dairy products & 0.53 & $27 \cdot 1$ \\
\hline & Nuts & 0.50 & \\
\hline & Eggs & 0.50 & \\
\hline & Fish & 0.38 & \\
\hline & Soups & 0.32 & \\
\hline & Fruits & 0.27 & \\
\hline & Processed meat & -0.30 & \\
\hline & Sugar-sweetened beverages & -0.37 & \\
\hline & Processed vegetables & -0.38 & \\
\hline \multirow[t]{5}{*}{ Sweets and seafood } & Cantonese desserts & 0.68 & $32 \cdot 9$ \\
\hline & Molluscs and shellfish & 0.62 & \\
\hline & Sugar-sweetened beverages & 0.36 & \\
\hline & Grains & -0.29 & \\
\hline & Leafy and cruciferous vegetables & -0.34 & \\
\hline
\end{tabular}

*For simplicity, factor loadings $< \pm 0.25$ were not listed.

In addition, a high prudent pattern score was associated with a slightly older age, higher education level and less parity, while a high sweets and seafood pattern score was associated with a higher income level and more parity (Table 2).

\section{Multivariate regression analysis}

Among the 3063 pregnant women, 544 (17.8\%) were diagnosed with GDM. Table 3 summarises the multivariate regression analysis for the associations between the tertiles of dietary pattern scores and the risk of GDM. After adjustment for maternal age, education level, monthly income, parity, pre-pregnancy BMI and family history of diabetes, the highest tertile (tertile 3 ) of the vegetable pattern was associated with a decreased risk of GDM (RR 0.79, 95\% CI 0.64, 0.97, $P$ for trend $=0.036$ ) compared with the lowest tertile (tertile 1 ; Table 3), whereas the third highest tertile of the sweets and seafood pattern was associated with an increased risk of GDM (RR $1 \cdot 23,95 \%$ CI $1 \cdot 02,1 \cdot 49, P$ for trend=0.010) compared with the lowest tertile (Table 3 ). In contrast, we found no significant association in the adjusted analysis for either the protein-rich or the prudent pattern (Table 3).

\section{Stratified analysis}

In the stratified analyses, there were no modification effects for the protein-rich, prudent, and sweets and seafood patterns by maternal age, education level, monthly income, prepregnancy BMI and parity ( $P$ for interaction $>0.05$, for all). However, the protective effect of a high vegetable pattern score appeared to be more evident among women who had a family history of diabetes (Fig. 1). Compared with the lowest tertile of the vegetable pattern score, the RR of the highest tertile were 0.94 (95\% CI $0.74,1.19)$ and 0.49 (95\% CI $0.31,0.76)$ for women with no family history of diabetes and those with a family history of diabetes, respectively ( $P$ for interaction $=0.022$; Fig. 1 ).

\section{Discussion}

In the present large prospective study, we investigated the associations between dietary patterns during pregnancy and the risk of GDM, and found that the vegetable dietary pattern was significantly associated with a decreased risk of GDM. In contrast, the sweets and seafood pattern was positively associated with the risk of GDM. To our knowledge, this is the first 
Table 2. Distributions of maternal characteristics by dietary pattern score tertiles in 3063 pregnant women from the Born in Guangzhou Cohort Study (Mean values and standard deviations; number of participants and percentages)

\begin{tabular}{|c|c|c|c|c|c|c|c|c|c|c|c|c|c|c|c|c|c|c|}
\hline \multirow[b]{3}{*}{ Characteristics } & & & \multicolumn{4}{|c|}{ Vegetable pattern* } & \multicolumn{4}{|c|}{ Protein-rich pattern* } & \multicolumn{4}{|c|}{ Prudent pattern* } & \multicolumn{4}{|c|}{ Sweets and seafood pattern ${ }^{\star}$} \\
\hline & \multicolumn{2}{|c|}{ All participants } & \multicolumn{2}{|c|}{ Tertile 1} & \multicolumn{2}{|c|}{ Tertile 3} & \multicolumn{2}{|c|}{ Tertile 1} & \multicolumn{2}{|c|}{ Tertile 3} & \multicolumn{2}{|c|}{ Tertile 1} & \multicolumn{2}{|c|}{ Tertile 3} & \multicolumn{2}{|c|}{ Tertile 1} & \multicolumn{2}{|c|}{ Tertile 3} \\
\hline & $n$ & $\%$ & $n$ & $\%$ & $n$ & $\%$ & $n$ & $\%$ & $n$ & $\%$ & $n$ & $\%$ & $n$ & $\%$ & $n$ & $\%$ & $n$ & $\%$ \\
\hline \multicolumn{19}{|l|}{ Age (years) } \\
\hline Mean & \multirow{3}{*}{\multicolumn{2}{|c|}{$\begin{array}{c}28 \cdot 9 \\
3 \cdot 2\end{array}$}} & \multicolumn{2}{|c|}{28.7} & \multirow{2}{*}{\multicolumn{2}{|c|}{$28 \cdot 9$}} & & & \multirow{2}{*}{\multicolumn{2}{|c|}{$\begin{array}{c}28.7 \\
3.1\end{array}$}} & & & \multirow{2}{*}{\multicolumn{2}{|c|}{$\begin{array}{c}29.1 \\
3.4\end{array}$}} & \multirow{2}{*}{\multicolumn{2}{|c|}{$28 \cdot 9$}} & \multicolumn{2}{|c|}{29} \\
\hline $\mathrm{SD}$ & & & & & & & & & & & & & & & & & & \\
\hline$P \dagger$ & & & \multicolumn{2}{|c|}{0.266} & & & \multicolumn{2}{|c|}{$\begin{array}{l}3.4 \\
0.002\end{array}$} & & & & & & & & & & \\
\hline Education level & & & & & & & & & & & & & & & & & & \\
\hline Middle school or & 306 & 10 & 121 & 11.9 & 85 & 8.3 & 106 & $10 \cdot 4$ & 108 & $10 \cdot 6$ & 133 & 13.0 & 75 & $7 \cdot 3$ & 111 & $10 \cdot 9$ & 98 & 9.6 \\
\hline College & 756 & 24.7 & 292 & 28.6 & 217 & 21.3 & 235 & 23.0 & 261 & $25 \cdot 6$ & 281 & $27 \cdot 5$ & 223 & 21.8 & 245 & $24 \cdot 0$ & 253 & $24 \cdot 8$ \\
\hline Undergraduate & 1637 & 53.4 & 545 & 53.4 & 541 & 53.0 & 526 & 51.5 & 566 & 55.4 & 485 & 47.5 & 592 & 58.0 & 537 & $52 \cdot 6$ & 549 & $53 \cdot 8$ \\
\hline Postgraduate & 364 & 11.9 & 63 & $6 \cdot 2$ & 178 & $17 \cdot 4$ & 154 & $15 \cdot 1$ & 86 & 8.4 & 122 & 11.9 & 131 & $12 \cdot 8$ & 128 & $12 \cdot 5$ & 121 & 11.9 \\
\hline$P+$ & & & & & & & & & & & & & & & & & & \\
\hline Monthly income $(Y$ & & & & & & & & & & & & & & & & & & \\
\hline$<1500$ & 227 & 8.2 & 76 & $8 \cdot 1$ & 54 & 5.9 & 75 & $8 \cdot 1$ & 78 & 8.4 & 80 & 8.7 & 66 & $7 \cdot 1$ & 82 & $9 \cdot 0$ & 72 & 7.6 \\
\hline $1500-4500$ & 1038 & 37.4 & 433 & $46 \cdot 2$ & 287 & 31.3 & 317 & 34.4 & 354 & 38.3 & 348 & 37.9 & 348 & 37.3 & 346 & 38.0 & 325 & $34 \cdot 2$ \\
\hline $4501-9000$ & 1120 & 40.4 & 334 & 35.6 & 417 & $45 \cdot 4$ & 379 & 41.1 & 380 & $41 \cdot 1$ & 366 & 39.9 & 377 & 40.4 & 373 & 41.0 & 393 & 41.4 \\
\hline$\geq 9001$ & 387 & 14 & 95 & $10 \cdot 1$ & 160 & $17 \cdot 4$ & 151 & $16 \cdot 4$ & 113 & $12 \cdot 2$ & 123 & 13.4 & 142 & $15 \cdot 2$ & 109 & $12 \cdot 0$ & 159 & $16 \cdot 8$ \\
\hline$P \dagger$ & & & & & & & & & & & & & & & & & & \\
\hline Pre-pregnancy BM & & & & & & & & & & & & & & & & & & \\
\hline$<18.5$ & 690 & 24.8 & 247 & $26 \cdot 2$ & 220 & 23.8 & 211 & 23.0 & 238 & $25 \cdot 8$ & 221 & 24.2 & 236 & $25 \cdot 3$ & 241 & $26 \cdot 4$ & 218 & $22 \cdot 9$ \\
\hline $18.5-23.9$ & 1844 & $66 \cdot 4$ & 616 & 65.4 & 633 & 68.4 & 623 & $67 \cdot 8$ & 604 & 65.4 & 600 & $65 \cdot 6$ & 627 & $67 \cdot 1$ & 582 & 63.7 & 650 & $68 \cdot 3$ \\
\hline$\geq 24$ & 244 & 8.8 & 79 & 8.4 & 73 & 7.9 & 85 & $9 \cdot 2$ & 82 & 8.9 & 94 & $10 \cdot 3$ & 71 & $7 \cdot 6$ & 90 & 9.9 & 84 & 8.8 \\
\hline$P+$ & & & & & & & & & & & & & & & & & & \\
\hline Family history of di & & & & & & & & & & & & & & & & & & \\
\hline No & 2274 & 80.6 & 769 & $80 \cdot 3$ & 748 & 79.7 & 770 & 81.9 & 755 & 80.7 & 741 & $79 \cdot 4$ & 756 & 79.9 & 763 & $82 \cdot 0$ & 770 & $79 \cdot 6$ \\
\hline Yes & 549 & $19 \cdot 4$ & 189 & $19 \cdot 7$ & 190 & $20 \cdot 3$ & 170 & $18 \cdot 1$ & 181 & $19 \cdot 3$ & 192 & $20 \cdot 6$ & 190 & $20 \cdot 1$ & 167 & 18.0 & 197 & 20.4 \\
\hline$P \dagger$ & & & & & & & & & & & & & & & & & & \\
\hline Parity $\ddagger$ & & & & & & & & & & & & & & & & & & \\
\hline 0 & 2639 & 93.3 & 889 & 92.6 & 871 & 92.8 & 899 & 95.4 & 860 & 91.6 & 845 & 90.5 & 906 & 95.5 & 875 & 93.8 & 881 & $91 \cdot 1$ \\
\hline$\geq 1$ & 190 & 6.7 & 71 & 7.4 & 68 & $7 \cdot 2$ & 43 & 4.6 & 79 & 8.4 & 89 & 9.5 & 43 & 4.5 & 58 & $6 \cdot 2$ & 86 & 8.9 \\
\hline$P+$ & & & & & & & & & & & & & & & & & & \\
\hline
\end{tabular}

* For simplicity, the numbers for tertile 2 are not listed.

TANOVA and $\chi^{2}$ tests were used to test the overall associations between maternal characteristics and three tertiles of dietary pattern scores.

¥ The number of missing values for monthly income, pre-pregnancy BMI, family history of diabetes and parity was $291,285,240$ and 234 , respectively. 
Table 3. Associations between the dietary patterns and the risk of gestational diabetes mellitus (GDM)

(Relative risks (RR) and 95\% confidence intervals)

\begin{tabular}{|c|c|c|c|c|c|c|}
\hline \multirow[b]{2}{*}{ Dietary patterns } & \multicolumn{2}{|c|}{ GDM cases ${ }^{*}$} & \multicolumn{2}{|c|}{ Model 1† } & \multicolumn{2}{|c|}{ Model $2 \ddagger$} \\
\hline & $n$ & $\%$ & $\mathrm{RR} \S$ & $95 \% \mathrm{Cl}$ & $\mathrm{RR} \S$ & $95 \% \mathrm{Cl}$ \\
\hline Total & 544 & $17 \cdot 8$ & & & & \\
\hline \multicolumn{7}{|l|}{ Vegetable } \\
\hline Tertile 1 & 192 & $18 \cdot 8$ & 1.00 & Reference & 1.00 & Reference \\
\hline Tertile 2 & 194 & $19 \cdot 0$ & 1.01 & $0.84,1.20$ & 1.00 & $0.83,1.21$ \\
\hline Tertile 3 & 158 & $15 \cdot 5$ & 0.81 & $0.67,0.99$ & 0.79 & $0.64,0.97$ \\
\hline$P$ for trend & & & & 0.047 & & 0.036 \\
\hline \multicolumn{7}{|l|}{ Protein-rich } \\
\hline Tertile 1 & 181 & $17 \cdot 7$ & 1.00 & Reference & 1.00 & Reference \\
\hline Tertile 2 & 183 & $17 \cdot 9$ & 1.01 & $0.83,1.21$ & 0.99 & $0.81,1.20$ \\
\hline Tertile 3 & 180 & $17 \cdot 6$ & 0.98 & $0.82,1.19$ & 0.95 & $0.78,1.16$ \\
\hline$P$ for trend & & & & 0.887 & & 0.659 \\
\hline \multicolumn{7}{|l|}{ Prudent } \\
\hline Tertile 1 & 181 & $17 \cdot 7$ & 1.00 & Reference & 1.00 & Reference \\
\hline Tertile 2 & 175 & $17 \cdot 1$ & 0.98 & $0.81,1.18$ & 0.96 & $0.78,1.17$ \\
\hline Tertile 3 & 188 & 18.4 & 1.04 & $0.87,1.25$ & 1.00 & $0.82,1.22$ \\
\hline$P$ for trend & & & & 0.719 & & 0.914 \\
\hline \multicolumn{7}{|c|}{ Sweets and seafood } \\
\hline Tertile 1 & 173 & $16 \cdot 9$ & 1.00 & Reference & 1.00 & Reference \\
\hline Tertile 2 & 156 & $15 \cdot 3$ & 0.88 & $0.73,1.08$ & 0.84 & $0.68,1.04$ \\
\hline Tertile 3 & 215 & $21 \cdot 1$ & 1.23 & $1.03,1.48$ & 1.23 & $1.02,1.49$ \\
\hline$P$ for trend & & & & 0.010 & & 0.010 \\
\hline
\end{tabular}

* Percentage of GDM in each third.

† Adjusted for other dietary patterns.

$\ddagger$ Adjusted for model 1 and for maternal age, education level, monthly income, pre-pregnancy BMI, family history of diabetes and parity.

$\S \mathrm{RR}(95 \% \mathrm{Cl})$ were derived by log-binomial regression models.

study to specifically identify the effects of dietary patterns on the risk of GDM in a Chinese population, which has distinct dietary habits compared with the Western population. Although there was a lack of direct evidence for dietary changes after conception in China, women are advised to follow a set of dietary customs, including avoidance of certain foods such as mutton and increasing consumption of special foods such as soup and fat during pregnancy according to traditional Chinese culture ${ }^{(23,24)}$. However, there is a lack of scientific justification for these dietary customs ${ }^{(23,24)}$.

Only a few studies have examined the effects of dietary patterns on the risk of $\mathrm{GDM}^{(11,15-17,25)}$. Of these studies, three were from a large prospective study, the Nurses' Health Study $\mathrm{II}^{(16,17,25)}$. Among these three studies, the one by Zhang et $a l{ }^{(16)}$ reported that the Western dietary pattern score was positively associated with the risk of GDM, whereas the prudent dietary pattern score was inversely associated. The prudent dietary pattern consisted of a high consumption of fruit, green leafy vegetables, poultry and fish, while the Western pattern represented a high consumption of red meat, processed meat, refined grain products, sweets, French fries and pizza ${ }^{(16)}$. Tobias et al. ${ }^{(25)}$ reported that healthy dietary patterns (alternate Mediterranean, Dietary Approaches to Stop Hypertension and alternate Healthy Eating Index) were significantly associated with a lower risk of GDM. Bao et $a l .{ }^{(17)}$ found that a pre-pregnancy lowcarbohydrate dietary pattern with high protein and fat from animal food sources was positively associated with the risk of GDM, whereas no association was found for a pre-pregnancy low-carbohydrate dietary pattern with high protein and fat from vegetable food sources. Another study from Mediterranean countries also observed that the Mediterranean diet was associated with a lower incidence of $\mathrm{GDM}^{(15)}$. However, a study from Massachusetts, USA did not find an association between dietary patterns during early pregnancy and the risk of $\mathrm{GDM}^{(11)}$.

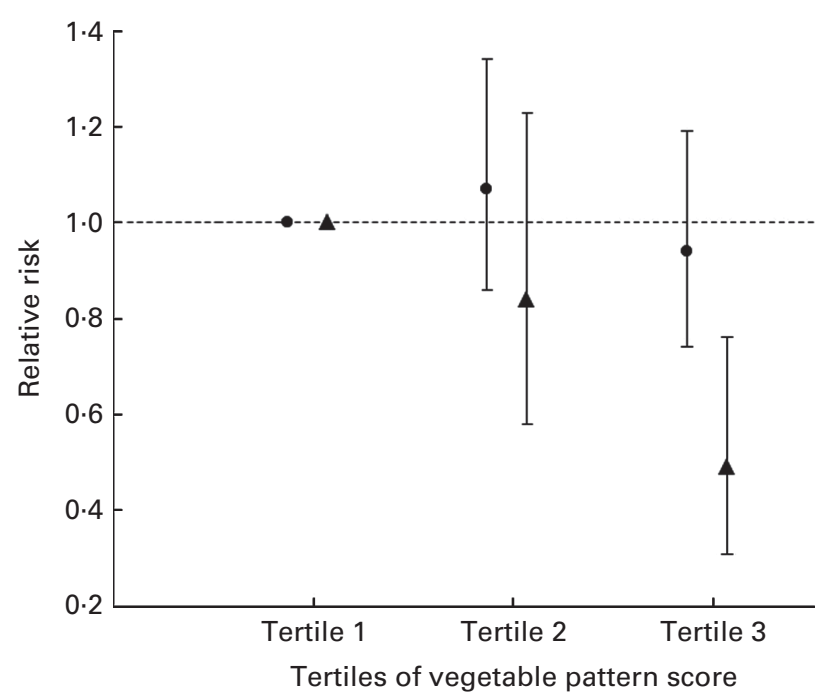

Fig. 1. Associations between vegetable pattern score tertiles and the risk of gestational diabetes mellitus, stratified by maternal family history of diabetes. $P$ for interaction $=0.022$. $\bullet$, Women without a family history of diabetes; $\mathbf{\Lambda}$, women with a family history of diabetes. 
In the present study, we observed that the vegetable dietary pattern was significantly associated with a decreased risk of GDM. This is in line with previous studies, in which vegetables and fruits were important components of protective dietary patterns ${ }^{(16,17,25)}$. In addition, there is evidence that dietary fibre was significantly associated with a decreased risk of $\mathrm{GDM}^{(12,26)}$. The association between dietary fibre patterns and the risk of GDM might be explained by some potential mechanisms. For instance, high dietary fibre intake may reduce appetite and food consumption, then reduce adiposity and improve insulin sensitivity ${ }^{(12,27)}$. Another possible mechanism might be that fibre intake could delay gastric emptying, and slow food digestion and absorption, thereby decreasing glucose absorption and plasma insulin levels ${ }^{(12,27,28)}$. Finally, SCFA, derived from colonic fermentation of indigestible dietary fibre, have been shown to improve lipid homeostasis, hence increasing insulin sensitivity ${ }^{(29,30)}$.

Family history of diabetes is a well-established risk factor for the development of type 2 diabetes as well as for $\mathrm{GDM}^{(31)}$ The underlying mechanisms might involve environmental factors that cluster within a family and genetic components that are shared among relatives. In addition, as a specific complication occurring during pregnancy, the risk of GDM could be affected not only by the maternal genome, but also by the fetal and paternal genomes ${ }^{(32-34)}$. In the present study, we observed that the protective effects of a high vegetable pattern score were more evident among women who had a family history of diabetes, including the family history of their partners. In contrast, previous studies have also reported significant interactions between family history of diabetes and other known risk factors, including obesity and occupational physical activity, on the risk of type 2 diabetes ${ }^{(35-37)}$. To our knowledge, the present study is the first to report on the effect of the interaction between dietary factors and family history of diabetes on the risk of diabetes. We speculated that the harmful effects of other risk factors of GDM, along with family history of diabetes, might be attenuated by the effects of a high vegetable pattern score. Although the underlying mechanism remains to be elucidated, this finding may have a significant implication for public health, as interventions focusing on women with a family history of diabetes may be highly effective.

We also found that the sweets and seafood pattern was associated with an increased risk of GDM. In the Nurses' Health Study II, a high intake of sugar-sweetened cola, but not other sugar-sweetened beverages, was associated with an increased risk of $\mathrm{GDM}^{(38)}$. These findings might be explained by the evidence that a high-sugar diet can decrease insulin sensitivity and insulin secretion ${ }^{(6,39,40)}$.

The protein-rich pattern was not associated with the risk of GDM, although previous studies have shown that a high intake of animal protein (especially red meat) was significantly associated with an elevated risk of $\mathrm{GDM}^{(13,16)}$. In addition, we did not find a significant effect of the prudent pattern on the development of GDM. Nevertheless, there is some epidemiological evidence suggesting that a high consumption of dairy products may be protective for type 2 diabetes and other components of the metabolic syndrome ${ }^{(41)}$. It has also been reported that a higher intake of nuts was associated with a significantly lower risk $^{(13)}$. Since dietary habits are population-specific, differences in study findings might be partly explained by differences in race/ethnicity between study populations.

The present study had several strengths, including the prospective design and a relatively large sample size. Compared with the analysis of individual nutrients or food items, dietary pattern analysis is able to capture synergistic effects among various nutrients and food, and the findings from dietary pattern analysis might be more comprehensive and informative for clinical practice and public health. However, there are also several limitations. First, we did not collect data on portion sizes of food items, and were unable to calculate the amount of food consumption. As previous studies have reported $^{(42-45)}$, frequencies of food intake were used as a proxy for a quantitative indicator in the present study. Thus, misclassification of exposure is inevitable. Such sources of error would bias results towards the null; hence, the strength of the observed associations might be underestimated. Second, we assessed food intake 'in the past week'. The information during this short period might not be representative of dietary habits throughout early pregnancy. However, previous studies have suggested that overall dietary patterns remain stable during pregnancy ${ }^{(46,47)}$. Third, dietary consumption was based only on the frequency of food intake and information on portion size was not collected in the present study; thus, we were unable to adjust for total energy intake. Finally, residual confounding, which is common in observational studies, cannot be excluded, although we adjusted for several factors in the statistical analysis.

In conclusion, we found that the vegetable dietary pattern was significantly associated with a decreased risk of GDM, while the sweets and seafood pattern was positively associated with the risk of GDM. These findings provide evidence to support the recommendation to eat a balanced diet for pregnant women in the Chinese population. Further studies are needed to clarify the associations between dietary patterns and the risk of GDM as well as pregnancy outcomes in other Asian populations.

\section{Supplementary material}

To view supplementary material for this article, please visit http://dx.doi.org/10.1017/S0007114515000707

\section{Acknowledgements}

The authors are grateful to the pregnant women who participated in the BIGCS and all obstetric care providers who assisted in the implementation of the study.

The present study was supported by the grants from the Guangzhou Science and Technology Bureau, Guangzhou, China (grant no. 2011Y2-00025 and 2012J5100038).

The authors' contributions are as follows: H.-M. X. and X. Q. conceived and designed the study; J.-R. H. and M.-Y. Y. conceived and designed the study, analysed and interpreted the data, and drafted the manuscript; N.-N. C., J.-H. L. and Y. L. 
analysed and interpreted the data; C.-Y. H., W.-B. M., R.-F. Z., Y.-H. P., L. Q., Y.-F. W. and W.-Q. X. collected and assembled the data. All authors performed critical revision for important intellectual content and approved the final version of the manuscript.

There are no conflicts of interest.

\section{References}

1. Reece EA, Leguizamon G \& Wiznitzer A (2009) Gestational diabetes: the need for a common ground. Lancet 373, 1789-1797.

2. American Diabetes Association (2004) Gestational diabetes mellitus. Diabetes Care 27, Suppl. 1, S88-S90.

3. Buckley BS, Harreiter J, Damm P, et al. (2012) Gestational diabetes mellitus in Europe: prevalence, current screening practice and barriers to screening. A review. Diabet Med 29, 844-854.

4. Dabelea D, Snell-Bergeon JK, Hartsfield CL, et al. (2005) Increasing prevalence of gestational diabetes mellitus (GDM) over time and by birth cohort: Kaiser Permanente of Colorado GDM Screening Program. Diabetes Care 28, 579-584.

5. Barbour LA (2014) Unresolved controversies in gestational diabetes: implications on maternal and infant health. Curr Opin Endocrinol Diabetes Obes 21, 264-270.

6. Zhang C \& Ning Y (2011) Effect of dietary and lifestyle factors on the risk of gestational diabetes: review of epidemiologic evidence. Am J Clin Nutr 94, 1975S-1979S.

7. Bo S, Menato G, Lezo A, et al. (2001) Dietary fat and gestational hyperglycaemia. Diabetologia 44, 972-978.

8. Gonzalez-Clemente JM, Carro O, Gallach I, et al. (2007) Increased cholesterol intake in women with gestational diabetes mellitus. Diabetes Metab 33, 25-29.

9. Qiu C, Frederick IO, Zhang C, et al. (2011) Risk of gestational diabetes mellitus in relation to maternal egg and cholesterol intake. Am J Epidemiol 173, 649-658.

10. Wang Y, Storlien LH, Jenkins AB, et al. (2000) Dietary variables and glucose tolerance in pregnancy. Diabetes Care 23, 460-464.

11. Radesky JS, Oken E, Rifas-Shiman SL, et al. (2008) Diet during early pregnancy and development of gestational diabetes. Paediatr Perinat Epidemiol 22, 47-59.

12. Zhang C, Liu S, Solomon CG, et al. (2006) Dietary fiber intake, dietary glycemic load, and the risk for gestational diabetes mellitus. Diabetes Care 29, 2223-2230.

13. Bao W, Bowers K, Tobias DK, et al. (2013) Prepregnancy dietary protein intake, major dietary protein sources, and the risk of gestational diabetes mellitus: a prospective cohort study. Diabetes Care 36, 2001-2008.

14. Hu FB (2002) Dietary pattern analysis: a new direction in nutritional epidemiology. Curr Opin Lipidol 13, 3-9.

15. Karamanos B, Thanopoulou A, Anastasiou E, et al. (2014) Relation of the Mediterranean diet with the incidence of gestational diabetes. Eur J Clin Nutr 68, 8-13.

16. Zhang C, Schulze MB, Solomon CG, et al. (2006) A prospective study of dietary patterns, meat intake and the risk of gestational diabetes mellitus. Diabetologia 49, 2604-2613.

17. Bao W, Bowers K, Tobias DK, et al. (2014) Prepregnancy low-carbohydrate dietary pattern and risk of gestational diabetes mellitus: a prospective cohort study. Am J Clin Nutr 99, 1378-1384.

18. Zhou B (2002) Prospective study for cut-off points of body mass index in Chinese adults. Zhonghua Liu Xing Bing Xue Za Zhi 23, 431-434.
19. Metzger BE, Gabbe SG, Persson B, et al. (2010) International association of diabetes and pregnancy study groups recommendations on the diagnosis and classification of hyperglycemia in pregnancy. Diabetes Care 33, 676-682.

20. Englund-Ogge L, Brantsaeter AL, Sengpiel V, et al. (2014) Maternal dietary patterns and preterm delivery: results from large prospective cohort study. BMJ 348, g1446.

21. McNutt LA, Wu C, Xue X, et al. (2003) Estimating the relative risk in cohort studies and clinical trials of common outcomes. Am J Epidemiol 157, 940-943.

22. Gruber HE, Rhyne AL 3rd, Hansen KJ, et al. (2012) Deleterious effects of discography radiocontrast solution on human annulus cell in vitro: changes in cell viability, proliferation, and apoptosis in exposed cells. Spine J 12, 329-335.

23. Gao H, Stiller CK, Scherbaum V, et al. (2013) Dietary intake and food habits of pregnant women residing in urban and rural areas of Deyang City, Sichuan Province, China. Nutrients 5, 2933-2954

24. Tian HG, Nan Y, Hu G, et al. (1996) A dietary survey of the Chinese population in urban and rural areas of Tianjin. Asia Pac J Clin Nutr 5, 229-232.

25. Tobias DK, Zhang C, Chavarro J, et al. (2012) Prepregnancy adherence to dietary patterns and lower risk of gestational diabetes mellitus. Am J Clin Nutr 96, 289-295.

26. Tovar A, Must A, Bermudez OI, et al. (2009) The impact of gestational weight gain and diet on abnormal glucose tolerance during pregnancy in Hispanic women. Matern Child Health J 13, 520-530.

27. McIntosh M \& Miller C (2001) A diet containing food rich in soluble and insoluble fiber improves glycemic control and reduces hyperlipidemia among patients with type 2 diabetes mellitus. Nutr Rev 59, 52-55.

28. Feskens EJ \& Kromhout D (1990) Habitual dietary intake and glucose tolerance in euglycaemic men: the Zutphen Study. Int I Epidemiol 19, 953-959.

29. Weickert MO \& Pfeiffer AF (2008) Metabolic effects of dietary fiber consumption and prevention of diabetes. J Nutr $\mathbf{1 3 8}$, 439-442.

30. Galisteo M, Duarte J \& Zarzuelo A (2008) Effects of dietary fibers on disturbances clustered in the metabolic syndrome. J Nutr Biochem 19, 71-84.

31. Ben-Haroush A, Yogev Y \& Hod M (2004) Epidemiology of gestational diabetes mellitus and its association with type 2 diabetes. Diabet Med 21, 103-113.

32. Petry CJ, Ong KK \& Dunger DB (2007) Does the fetal genotype affect maternal physiology during pregnancy? Trends Mol Med 13, 414-421.

33. Petry CJ, Seear RV, Wingate DL, et al. (2011) Associations between paternally transmitted fetal IGF2 variants and maternal circulating glucose concentrations in pregnancy. Diabetes 60, 3090-3096.

34. Zhang C, Bao W, Rong Y, et al. (2013) Genetic variants and the risk of gestational diabetes mellitus: a systematic review. Hum Reprod Update 19, 376-390.

35. Harrison TA, Hindorff LA, Kim H, et al. (2003) Family history of diabetes as a potential public health tool. Am J Prev Med 24, 152-159.

36. Sargeant LA, Wareham NJ \& Khaw KT (2000) Family history of diabetes identifies a group at increased risk for the metabolic consequences of obesity and physical inactivity in EPIC-Norfolk: a population-based study. The European Prospective Investigation into Cancer. Int $J$ Obes Relat Metab Disord 24, 1333-1339.

37. Morris RD, Rimm DL, Hartz AJ, et al. (1989) Obesity and heredity in the etiology of non-insulin-dependent diabetes 
mellitus in 32,662 adult white women. Am J Epidemiol 130 112-121.

38. Chen L, Hu FB, Yeung E, et al. (2009) Prospective study of pregravid sugar-sweetened beverage consumption and the risk of gestational diabetes mellitus. Diabetes Care 32, 2236-2241.

39. Daly M (2003) Sugars, insulin sensitivity, and the postprandial state. Am J Clin Nutr 78, 865S-872S

40. Davis JN, Alexander KE, Ventura EE, et al. (2007) Associations of dietary sugar and glycemic index with adiposity and insulin dynamics in overweight Latino youth. Am J Clin Nutr 86, 1331-1338.

41. Turner KM, Keogh JB \& Clifton PM (2015) Dairy consumption and insulin sensitivity: a systematic review of short- and longterm intervention studies. Nutr Metab Cardiovasc Dis 25, 3-8.

42. Perrine CG, Galuska DA, Thompson FE, et al. (2014) Breastfeeding duration is associated with child diet at 6 years. Pediatrics 134, Suppl. 1, S50-S55.
43. Park S, Pan L, Sherry B, et al. (2014) The association of sugar-sweetened beverage intake during infancy with sugarsweetened beverage intake at 6 years of age. Pediatrics $\mathbf{1 3 4}$, Suppl. 1, S56-S62.

44. Braga DP, Halpern G, Figueira Rde C, et al. (2012) Food intake and social habits in male patients and its relationship to intracytoplasmic sperm injection outcomes. Fertil Steril 97, 53-59.

45. Dahlquist GG, Blom LG, Persson LA, et al. (1990) Dietary factors and the risk of developing insulin dependent diabetes in childhood. BMJ 300, 1302-1306.

46. Cuco G, Fernandez-Ballart J, Sala J, et al. (2006) Dietary patterns and associated lifestyles in preconception, pregnancy and postpartum. Eur J Clin Nutr 60, 364-371.

47. Crozier SR, Robinson SM, Godfrey KM, et al. (2009) Women's dietary patterns change little from before to during pregnancy. J Nutr 139, 1956-1963. 